\title{
Henry E. Sigerist: Die Gestaltung des medizinhistorischen Unterrichts
}

\section{Ein unpubliziertes Manuskript im Zürcher Medizinhistorischen Institut}

herausgegeben von Christoph Mörgeli und André Blaser

\section{ZuSAMMENFASSUNG}

Henry E.Sigerist (1891-1957) verfaßte 1951 eine bisher unveröffentlichte Abhandlung über die Gestaltung des medizinhistorischen Unterrichts. Hierbei stützte er sich auf eigene jahrelange Erfahrungen in Leipzig und Baltimore. Seiner Meinung nach mu $\beta$ eine lebendige Medizingeschichte auf die aktuelle Medizin Bezug nehmen. Darüber hinaus sollte sie versuchen, "die Kluft zwischen Geistes-, Sozial- und Naturwissenschaften» zu überbrücken. Henry E. Sigerists Vorschlag für einen möglichen Lehrplan umfaßt eine Einführungsvorlesung in die Medizin für Anfänger und eine Vorlesung über Geschichte der Medizin für vorgerücktere Studenten. Vorlesungen über ärztliche Ethik und Soziologie der Medizin sowie auch Seminar-Übungen und ein Kolloquium zur weiteren Vertiefung besonderer Gebiete sind zusätzliche Bestandteile seines Lehrplans.

\section{Zur Entstehung des Manuskripts}

Ein Teil des wissenschaftlichen Nachlasses von Henry E.Sigerist (1891-1957) wird im Medizinhistorischen Institut der Universität Zürich aufbewahrt. Unter Sigerists Manuskriptheften fand sich eine bisher unveröffentlichte Schrift zur Gestaltung des medizinhistorischen Unterrichts. Der Aufsatz ist am 11. Februar 1951 in Pura (Tessin) entstanden und umfaßt die ersten siebzehn Seiten eines unlinierten Notizheftes $(33 \times 21 \mathrm{~cm})$; außerdem befinden sich im Manuskriptheft weitere sechs handschriftliche Miszellen von geringerem Umfang, die aber alle auch gedruckt vorliegen.

Datierung und Thematik lassen es als naheliegend erscheinen, daß die hier geäußerten Gedanken Sigerists zum medizinhistorischen Unterricht in direktem Zusammenhang mit der Errichtung des ersten vollamtlichen 
medizinhistorischen Lehrstuhls in der Schweiz, nämlich in Zürich, stehen. Kurz davor hatte der Regierungsrat des Kantons Zürich nämlich einem entsprechenden Antrag der Medizinischen Fakultät zugestimmt und ein Medizinhistorisches Institut geschaffen; dessen Direktor sollte als Extraordinarius an der Universität über Medizingeschichte lesen und gleichzeitig als Konservator die von PD Dr. Gustav Adolf Wehrli (1888-1949) zusammengetragene, 1932 vom Kanton übernommene Sammlung betreuen. Damit war die Forderung der Schaffung eines Medizinhistorischen Instituts - seit Sigerists Habilitation in Zürich (1921) in periodischen Abständen immer wieder diskutiert - erfüllt worden. Henry E. Sigerist, inzwischen weltweit führender Medizinhistoriker in Leipzig (1925-1932) und Baltimore (bis 1947), hatte sich mehrmals entschieden für die Schaffung eines entsprechenden Lehrstuhls und Besetzung desselben durch eine erste Kraft ausgesprochen. Sigerist selbst stand nach der Übersiedlung von den Vereinigten Staaten in die Schweiz vorübergehend als Kandidat für die neue Zürcher Professur im Gespräch. 1949 beurteilte er in einem Gutachten PD Dr. Bernhard Milt (1896-1956) als den derzeit bestqualifizierten Schweizer Medizinhistoriker (Dekan Hans Fischer an Erziehungsdirektion, 13.7.1949). Milt wurde auf Frühjahr 1951 denn auch zum ersten Vorsteher des Zürcher Medizinhistorischen Instituts gewählt.

Sigerists Vorschläge zur Gestaltung des medizinhistorischen Unterrichts dürften sich an die Adresse Milts gerichtet haben. Den einschlägigen Akten und Protokollen läßt sich nicht entnehmen, daß es sich dabei um einen offiziellen Gutachterauftrag von Regierung oder Fakultät im Hinblick auf die Aufnahme von Milts Lehrtätigkeit gehandelt hätte. Andererseits geht Sigerists Schrift über einen brieflich niedergelegten kollegialen Ratschlag hinaus. Es scheint uns denkbar, daß Sigerist sein Manuskript 1951 den Teilnehmern der «Pura-Konferenz» vorgetragen hat, also jenem medizinhistorisch interessierten Kreis, der sich alljährlich in Sigerists Tessiner Heim in wissenschaftlich anregender und geselliger Atmosphäre um den Meister versammelte.

Zwei weitere Male hat Henry E. Sigerist über didaktische und methodische Fragen der Vermittlung des Fachs Medizingeschichte geschrieben. In einem Aufsatz von $1934 \mathrm{im}$ Bulletin seines Instituts an der Johns Hopkins University (vol.2, p. 123-140) äußerte er sich sehr detailliert zur Behandlung der einzelnen Stoffgebiete von der primitiven Heilkunde bis zum Aufstieg der amerikanischen Medizin. 1953 hielt er einen Vortrag vor dem Osler Club in London (The Practitioner 171, 1953, p. 188-194) mit dem Titel 
«Medical History in Medical Education». Dazu hat er den hier herausgegebenen Manuskripttext vollständig umgearbeitet, so daß im zwei Jahre später gehaltenen Referat nur noch vereinzelte Passagen anklingen. Er legte das Gewicht wiederum auf die ökologischen und soziologischen Aspekte der Medizingeschichte und setzte sich überdies kritisch mit der im Rahmen des Medizinstudiums zur Verfügung stehenden Zeit und dem Mangel an kompetenten Lehrern auseinander.

Die Ausführungen von Henry E. Sigerist über die Gestaltung des medizinhistorischen Unterrichts in der unmittelbaren Nachkriegszeit dürften auch heute noch von Interesse sein, hat Sigerist doch mit seiner konsequenten Einbettung der Medizingeschichte in die Kultur- und Sozialgeschichte neue, auch heute noch gültige Wege beschritten. Es war ihm ein wichtiges Anliegen, die künftigen Ärzte auf die großen historischen Zusammenhänge ihres Berufs aufmerksam zu machen; dabei hat er den Aufgabenkreis des Medizinhistorikers außerordentlich weit gesteckt, beanspruchte er doch die Einführung in die Medizin, die medizinische Ethik und die Soziologie der Medizin gleichermaßen für sein Fach. Seine Ratschläge verdienen um so mehr Beachtung, als Sigerist zum Zeitpunkt der Niederschrift auf über ein Vierteljahrhundert erfolgreicher eigener Tätigkeit als Hochschullehrer zurückblicken konnte.

\section{Sigerists Text}

Ich habe Geschichte der Medizin während sechsundzwanzig Jahren in einer Reihe von Ländern auf drei Kontinenten [Europa, Amerika, Asien] unterrichtet, so daß ich eine gewisse Erfahrung gesammelt habe. Ganz allgemein habe ich gefunden, daß die Studenten ausgezeichnet mitgingen und lebhaftes Interesse zeigten, sofern man den Gegenstand nicht rein antiquarisch vortrug, sondern Bezug nahm auf Probleme der lebendigen Medizin.

Sehr erfreulich fand ich auch, daß Geschichte der Medizin ein Brückenfach zwischen den Fakultäten bildet. In Zürich habe ich zusammen mit dem klassischen Philologen Prof. Ernst Howald Seminare abgehalten, in denen hippokratische und aristotelische Schriften im griechischen Text gelesen und interpretiert wurden und die von Studenten der Philologie und Medizin und sogar von einigen praktischen Ärzten besucht waren. In Leipzig habe ich mich öfters an Übungen des Instituts für Kultur- und Universalgeschichte beteiligt (z. B. Kritisch-historische Übungen über das Wunder; Übungen 
über Leonardo da Vinci u[nd] ä[hnlichen] I[nhalts]). Ich habe auch einmal im Ägyptologischen Institut zusammen mit dem Ägyptologen Wolf ein Seminar über einen ägyptischen medizinischen Papyrus (Edwin Smith) abgehalten. Andererseits haben Dozenten der Philosophischen Fakultät sich häufig an Seminaren meines Medizinhistorischen Instituts beteiligt. So erinnere ich mich an eine sehr gut gelungene Übung über das Zeitalter des Barock, in welcher Geschichte, Wirtschaft, Religion, Philosophie, Literatur, Kunst, Musik, Naturwissenschaft, Technik, Medizin und die Hauptkrankheiten der Periode zur Diskussion kamen und man am Schluß versuchte, den allen Äußerungen gemeinsamen Stil herauszuarbeiten.

In Baltimore habe ich nicht nur in der medizinischen Fakultät, sondern auch in der School of Hygiene and Public Health unterrichtet und gelegentlich im College of Arts and Sciences. In allen meinen Seminaren hatte ich neben Medizinstudenten auch solche der Geschichte, Nationalökonomie, Soziologie; an meinem soziologischen Abendseminar beteiligten sich regelmäßig auch Regierungsbeamte aus dem benachbarten Washington. In dieser Weise kann das Fach in sehr glücklicher Weise dazu beitragen, die Kluft zwischen Geistes-, Sozial- und Naturwissenschaften zu überbrükken.

Nun sind natürlich die Verhältnisse an jeder Universität etwas verschieden. Die Gestaltung des Lehrplans, die Zusammensetzung der Studentenschaft, die räumliche Lage der Institute, die ganze Atmosphäre wechseln von Ort zu Ort, und man muß erst ausfindig machen, wie weit man gehen und wieviel man bieten kann. Es scheint mir jedoch, daß gewisse grundlegende Vorlesungen überall mit Nutzen gegeben werden können, und ich würde folgende vorschlagen, die sich mir sehr bewährt haben:

\section{Einführung in die Medizin}

Eine Vorlesung für Anfänger, etwa Studenten des dritten Semesters, zweistündig im Winter-Semester. Zweck der Vorlesung, die auf breiter historisch-philosophischer Grundlage aufgebaut wird, ist, den angehenden Medizinern zu sagen, was Medizin ist, welches ihre Stellung im System der Wissenschaften und ihre Aufgaben in der Gesellschaft sind. Ich habe die Vorlesung in Leipzig regelmäßig gehalten, und mein Buch «Einführung in die Medizin» ist daraus hervorgegangen (Leipzig 1931), das in sechs Sprachen übersetzt wurde. In Baltimore habe ich die Vorlesung ab und zu 
Assistenten überlassen, um ihnen Gelegenheit zu geben, sich zu üben. Das letzte Mal, daß ich die Vorlesung hielt, hatte sie folgenden Aufbau:

I. Der gesunde Mensch

1. Der Mensch in der Natur

a. Bau

b. Funktion

c. Seele und Geist

2. Der Mensch in der Gesellschaft

II. Wert und Erhaltung der Gesundheit

III. Der kranke Mensch

IV. Die Krankheit

1. Die Zeichen der Krankheit

2. Das Wesen der Krankheit

3. Krankheiten

4. Vorkommen der Krankheiten

5. Soziale und wirtschaftliche Folgen der Krankheit

V. Ursachen der Krankheit

1. Vererbung

2. Soziale Umwelt

3. Physische Umwelt

\section{Das Verhüten der Krankheit}

VII. Die ärztliche Hilfe

1. Krankengeschichte

2. Diagnose und Prognose

3. Behandlung

4. Soziale Wiederẹingliederung

VIII. Der Arzt

Ich glaube nicht, daß eine solche Vorlesung anderen Fächern irgendwie vorgreift, da sie ja überaus allgemein und elementar gehalten werden muß. 


\section{Geschichte der Medizin}

Wohl am besten für etwas vorgerücktere Studenten, doch kann die Vorlesung von allen Semestern gehört werden. In Leipzig gab ich sie zwei Semester zweistündig; in Baltimore gab ich eine Übersichtsvorlesung von sechzehn Stunden, die ergänzt wurde durch sechs Stunden Geschichte der amerikanischen Medizin (von einem Assistenten gegeben) und durch Seminare. Am besten schiene es mir, man würde hier beginnen mit einer einsemestrigen zweistündigen Vorlesung.

In der «Einführung» liegt der Hauptakzent auf Medizin, und die historische Betrachtung ist Methode. Hier ist der Hauptakzent auf Geschichte, und es handelt sich darum, die Entwicklung der Medizin (im weitesten Sinne des Wortes) in ihren Hauptzügen darzustellen, zu zeigen, daß sie eine Teilerscheinung der allgemeinen Kultur eines Volkes ist. Auch läßt sich an Hand der Geschichte vieles illustrieren; die Besprechung der primitiven Medizin zeigt Anschauungen, die nie untergehen, sondern vom Arzt bei manchen Patienten angewendet werden. Die Babylonische Medizin ist ein religiöses System der Medizin, und man kann dabei zeigen, daß religiöse Medizin bei jeder Gesellschaft in irgendeiner Form vorkommt usw.

\section{3. Ärztliche Ethik}

In Leipzig gab ich regelmäßig eine solche Vorlesung einstündig, in welcher ich die drei Kreise, die das ärztliche Handeln bestimmen, besprach und historisch entwickelte: der Staat und seine Gesetze, die Ärzteschaft und ihre Normen, das individuelle Gewissen und die es bestimmenden Faktoren. In Baltimore gab ich die Vorlesung nicht, da ein anderes Mitglied der Fakultät einige Vorträge über dieses Thema zu halten pflegte. Ich glaube, daß eine solche Vorlesung durchaus wertvoll sein kann.

\section{Soziologie der Medizin}

Auf Wunsch der Fakultät und da ich auf diesem Gebiet viel gearbeitet hatte, habe ich in Baltimore regelmäßig Vorlesungen und Übungen zu diesem Thema gehalten, die stark besucht waren.

Die Vorlesung, wie ich sie zuletzt 1946-47 hielt, umfaßte zweiunddreißig 
Stunden und hatte folgenden Aufbau (ich gebe die Themen englisch wieder, da die Vorlesung auf amerikanische Verhältnisse zugeschnitten war): copy p. 138 [im Manuskript nicht vorhanden].

Die Themen wurden jeweils historisch und soziologisch entwickelt. Sollte eine solche Vorlesung erwünscht sein, so würde ich sie so umarbeiten, daß die schweizerischen und europäischen Verhältnisse in erster Linie berücksichtigt wären, ohne daß dabei die sehr interessanten amerikanischen Studien außer acht gelassen würden.

\section{Seminar- Übungen}

Wo ein Lehrstuhl für Geschichte der Medizin vorhanden ist, darf man sich nicht auf allgemeine Vorlesungen beschränken, sondern muß Studenten die Gelegenheit geben, tiefer in das Gebiet einzudringen und die speziellen Arbeitsmethoden der Medizingeschichte kennenzulernen. An allen Universitäten, an denen ich tätig war, habe ich stets historische Seminar-Übungen abgehalten über die verschiedensten Gebiete. Entweder wurden medizinische Klassiker-Texte gelesen und interpretiert oder es wurde ein besonderes Problem, wie z. B. der Konstitutionsbegriff, historisch-kritisch untersucht, oder eine Epoche wie die Romantik kam zur Sprache oder ein Gebiet wie die Entwicklung der Psychiatrie von Pinel zu Freud u.ä. Bei der Wahl der Themen habe ich mich jeweils von zwei Gesichtspunkten leiten lassen: von den Wünschen der Studenten und von der Arbeit, die mich selber jeweils beschäftigte. Ich habe nie eine größere Arbeit geschrieben, die ich nicht zuvor mit meinem Seminar besprochen hätte, um die Studenten am Forschungsprozeß teilnehmen zu lassen.

In Baltimore gab ich jedes Jahr ein soziologisch-medizinisches Seminar, das jeweils am Abend gehalten wurde und eine gewisse Berühmtheit erlangte. Etwa die Hälfte der Teilnehmer waren postgraduate students, d.h. junge Ärzte, die zur Fortbildung in Hygiene an der School of Hygiene studierten. Sechs Teilnehmer meines letzten Seminars von 1946-47 sind heute Professoren in angesehenen Stellungen. 


\section{Kolloquium}

In Leipzig habe ich regelmäßig, in Baltimore öfters wöchentliche Kolloquien abgehalten, in welchen Zeitfragen der Medizin in historisch-soziologischer Beleuchtung zur Diskussion kamen. Solche Kolloquien waren immer anregend und waren oft sehr stark besucht.

Zusammenfassend könnte ich mir einen Lehrplan vorstellen, der etwa folgendermaßen aussehen würde:

Winter-Semester:

Einführung in die Medizin

2 Stunden

Soziologie der Medizin

2 Stunden

Seminar

2 Stunden

Sommer-Semester:

Geschichte der Medizin

2 Stunden

Ärztliche Ethik

1 Stunde

Seminar

2 Stunden

Kolloquium

1 Stunde

Es sind alle Vorlesungen ihrem Wesen nach historisch, auch wenn das Wort «Geschichte» nicht in jedem Titel vorkommt, da ich die Themen jeweils historisch entwickle.

Natürlich dürfte ein solcher Plan nicht starr sein. Etliches bräuchte nicht jedes Jahr gelesen zu werden und an dessen Stelle könnte eine Spezialvorlesung treten. Soziologie der Medizin würde ich nur dann lesen, wenn die Fakultät es für wünschenswert hielte. Natürlich würde bei diesen Vorlesungen und Übungen auch viel davon abhängen, ob geeignete Stunden im Lehrplan gefunden werden könnten und wie die Studenten reagieren.

Pura, 11. Februar 1951 


\section{Summary}

\section{An unpublished manuscript by Henry E.Sigerist on the organization of teaching in medical history}

In 1951 Henry E. Sigerist (1891-1957) wrote a manuscript paper on the structure of lectures on medical history. In this paper, which has remained unpublished yet, Sigerist's considerations are based on many years of experience in teaching in Leipzig and Baltimore. According to him, a lively medical history must always refer to current medicine. Furthermore, it should try to bridge the gulf between the arts, the social and the natural sciences. His proposal for a possible schedule includes an introductory course of lectures into medicine for beginners and a course on the history of medicine for advanced students. Lectures on medical ethics and sociology of medicine are also part of his programme. Finally, Sigerist stresses the fact that, in addition to these four lecture courses, seminars and a colloquy may provide students with the possibility of working on a specific subject in more detail.

\section{Résumé}

Henry E. Sigerist: La forme de l'enseignement de l'histoire de la médecine. Un manuscrit inédit de l'Institut d'histoire de la médecine de Zurich

S'appuyant sur une longue expérience acquise à Leipzig et à Baltimore, Henry E. Sigerist (1891-1857) a écrit en 1951 une étude restée inédite sur la forme de l'enseignement de la médecine. Une histoire de la médecine qui soit vivante devrait se référer à la médecine actuelle et tenter de jeter un pont sur l'abîme qui sépare les études littéraires des sciences sociales et des sciences naturelles. L'auteur propose un programme d'enseignement qui comprend une leçon d'introduction à la médecine pour les débutants et une conférence sur l'histoire de la médecine pour étudiants avancés. Il prévoit également en complément des conférences sur l'éthique médicale et la sociologie de la médecine ainsi que des travaux de séminaires destinés à approfondir certains sujets.

Dr. phil. Christoph Mörgeli

Konservator des Medizinhistorischen Museums der Universität

Rämistraße 71

CH-8006 Zürich

cand.phil. André Blaser

Semesterassistent, Medizinhistorisches Institut

Rämistraße 71

CH-8006 Zürich 